Student's performance on the MAC was compared with their performance on their official university examination.

Test item analysis and psychometrics were also performed.

Results The passing score was determined at 13/30 (41.2\%).

366 undergraduate students sat the exam. Their mean score was $45.9 \%$ (s.d 9.9\%, range 23-73\%). 240/366 (65.4\%) of students achieved the passing score whereas $99 \%$ of these students passed their official university exam. However, there was a positive correlation between students result in the MAC exam and results from their official university exams (spearman $\mathrm{R}=0.44, \mathrm{p}<0.01$ ).

58 paediatric SHO's sat the exam. Their mean score was $64.2 \%$ (s.d $11.8 \%$, range $40-80 \%$ ) which was significantly better than undergraduate students $(\mathrm{p}<0.01)$.

After ranking test item difficulty, there was a positive correlation in responses between the students and the SHO's (spearman $\mathrm{R}=0.73(\mathrm{p}<0.01)$.

We identified a pattern of consistently poorly answered questions in order to highlight areas of a knowledge deficit. Conclusion This is a novel approach to paediatric assessment, enabling clinicians as opposed to academics to design the content.

The MAC exam is more difficult than expected and both students and SHO's found the same questions challenging.

Students results were significantly worse on the MAC compared with their official university exam. We need to explore further if this is due to a lack of knowledge (requiring curriculum change) or unreasonable expectations from clinicians.

The MAC exam showed reproducible results but other psychometric properties are not yet strong enough for it to be considered as a high stakes assessment tool in its current format.

\section{GP150 SUPERVISED LEARNING EVENTS: TRAINEES VIEW POINT}

Andy Thomson*, Pramodh Vallabhaneni. Department of Paediatrics,Morriston Hospital, Swansea, UK

\subsection{6/archdischild-2019-epa.214}

Introduction Supervised Learning Events (SLEs) were introduced into post graduate training to complement competency based medical education. SLEs were designed to have formative educational discussions between trainees and trainers. These involve checklists and judgments made on rating scales. This gives the process a subjective edge and can lead to trainees seeing them as merely tick box exercises for progression in training. We set out to evaluate Paediatric trainee perceptions of SLEs in Wales.

Methods An anonymous 10-item mixed methods cross sectional survey questionnaire was devised and distributed amongst Paediatric trainees in Wales. Ethical approval was obtained and data was collected over a two month period.

Results 24 trainees responded to the survey. Only 38\% respondents felt SLEs have positive educational value. Of the remaining 28\% felt SLEs weren't educationally productive and the rest had a neutral opinion. Difficulties in completing SLEs were a common theme. $62 \%$ felt assessors lacked time to do SLEs constructively. Finding a willing assessor in busy clinical areas was a common concern. Most trainees reported a summative attitude from trainers.
Conclusion The survey results are in line with the gathering evidence that SLEs need an urgent overhaul. For trainees to understand the educational value of SLEs there needs to be a change of direction from trainers. There is need for training at both levels to make SLEs more robust and multifaceted. Trainees in Wales have suggested how simple improvements can be made to enhance learning via SLEs.

\section{GP151 USING A MODIFIED-TOSBA APPROACH TO PROVIDE TIMELY, FORMATIVE FEEDBACK TO MEDICAL STUDENTS - 18 MONTHS EXPERIENCE IN TERTIARY PAEDIATRICS}

Daire O'Leary ${ }^{*}$, Leona Nertney, Alf Nicholson. RCSI Paediatric Dept, Dublin, Ireland

\subsection{6/archdischild-2019-epa.215}

Background Medical student numbers have increased, and medical degree course duration has decreased over recent years. This leads to reduced bedside teaching opportunities which has been implicated in a reduction in clinical skills. In addition, students' desire to receive formative feedback is well documented. Team objective structured bedside assessments (TOSBA) involves structured feedback on observed clinical tasks performed on patients in the small-group clinical tutorial setting.

The paediatric rotation in our institution includes six weeks of neonatal and paediatric hospital attachments. Students spend a third of their rotation in tertiary paediatric hospital. Each student is scheduled for two bedside tutorials with each clinical lecturer. In order to provide consistent formative feedback for all students, a decision was made to adopt a modified TOSBA approach to these learning experiences.

Methods A structure for the 60-minute mTOSBA bedside teaching sessions was agreed. Students were briefed on the structure of the session. Within each tutorial group, half the students were observed taking a focused history and the other half performing clinical examination. Tutors facilitated a verbal debrief immediately following the bedside session. In addition, each student received individualized, written, formative feedback. The emphasis was placed on providing formative feedback on communication and clinical skills rather than knowledge; in this way it differed from the original TOSBA described by Miller et al.

Results Over the first 18 months, almost 500 students have attended 1 or 2 mTOSBAs during their tertiary paediatric clinical rotation. Challenges encountered are typical for any bedside teaching session in terms of patient access and interruptions. Specific to mTOSBA, delays in providing written feedback and additional workload for clinical lecturers were noted. After the first year, written feedback was incorporated into the students' portfolio leading to more timely written feedback and reducing duplication of tasks by clinical lecturers. Verbal feedback from students on this mode of teaching has been positive.

Conclusions Formal evaluation of the acceptability and utility of mTOSBA as a tool for providing timely, formative feedback is ongoing. Our experience to date suggests that it is feasible even during a relatively short window with a relatively high student to lecturer ratio. 